\title{
The CMS Pixel Detector Upgrade and R\&D for the High Luminosity LHC
}

\author{
Lorenzo Viliani ${ }^{* \dagger}$ \\ INFN, Firenze (IT) \\ E-mail: lorenzo.vilianiecern.ch
}

The High Luminosity Large Hadron Collider (HL-LHC) at CERN is expected to collide protons at a centre-of-mass energy of $14 \mathrm{TeV}$ and to reach an unprecedented peak instantaneous luminosity of $5 \times 10^{34} \mathrm{~cm}^{-2} \mathrm{~s}^{-1}$ with an average number of pileup events of 140 . This will allow the ATLAS and CMS experiments to collect integrated luminosities of up to $3000 \mathrm{fb}^{-1}$ during the project lifetime. To cope with this extreme scenario the CMS detector will be substantially upgraded before starting the HL-LHC, a plan known as CMS Phase-2 Upgrade. In the upgrade the entire CMS silicon pixel detector will be replaced and the new detector will feature increased radiation hardness, higher granularity and capability to handle higher data rate and longer trigger latency. In this report the Phase-2 Upgrade of the CMS silicon pixel detector will be reviewed, focusing on the features of the detector layout and on the development of new pixel devices.

The 26th International Workshop on Vertex Detectors

10-15 September, 2017

Las Caldas, Asturias, Spain

\footnotetext{
${ }^{*}$ Speaker.

${ }^{\dagger}$ On behalf of the CMS Collaboration
} 


\section{Phase-2 Upgrade of the CMS Pixel detector}

Long Shutdown 3 (LS3) of the CERN LHC is scheduled to start in 2024 and last until 2026. It represents the period during which the accelerator and the experiments will be upgraded in view of the High Luminosity phase of the LHC, known as HL-LHC [1]. An overview of the data taking periods and shutdowns of the LHC until the high luminosity phase is shown in Fig. 1.

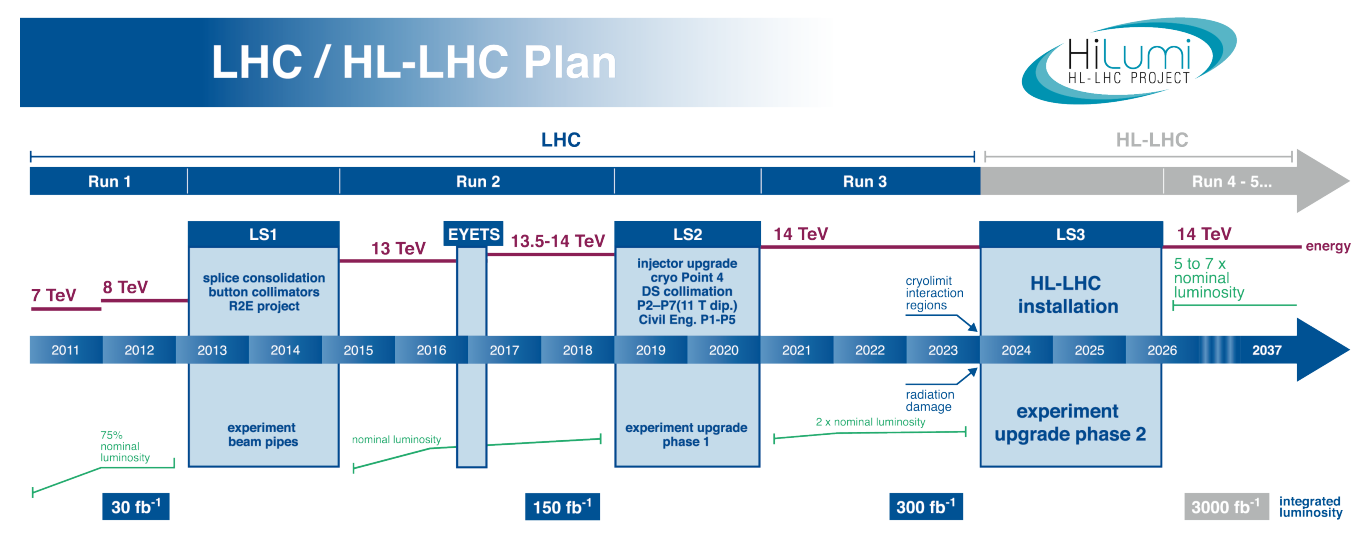

Figure 1: Overview of the LHC schedule up to the HL-LHC phase [1]. Data taking periods (Runs) are indicated, as well as the long shutdowns. Centre-of-mass energy and total integrated luminosity for different periods are shown. Also, the increase of the instantaneous luminosity with respect to the nominal value of $1 \times 10^{34} \mathrm{~cm}^{2} \mathrm{~s}^{-1}$ is reported.

The main goal of the LS3 accelerator upgrade is to achieve peak instantaneous luminosities of $5 \times 10^{34} \mathrm{~cm}^{2} \mathrm{~s}^{-1}$, or even $7.5 \times 10^{34} \mathrm{~cm}^{2} \mathrm{~s}^{-1}$ in the best performance scenario. The high instantaneous luminosity will allow the ATLAS and CMS experiments to collect an integrated luminosity up to $300 \mathrm{fb}^{-1}$ per year and up to $3000 \mathrm{fb}^{-1}\left(4000 \mathrm{fb}^{-1}\right.$ in the best performance scenario) during the HL-LHC operation time of ten years. The HL-LHC is expected to operate at a centre-of-mass energy of $14 \mathrm{TeV}$ and with a bunch crossing spacing of $25 \mathrm{~ns}$. The number of pileup events increases with the instantaneous luminosity and is expected to reach an average of 140 events, increasing to 200 in the best performance scenario. The radiation level in such a harsh scenario will be unprecedented, especially in the innermost layers of the detector, where a $1 \mathrm{MeV}$ neutron equivalent fluence of $2.3 \times 10^{16} \mathrm{n}_{\mathrm{eq}} / \mathrm{cm}^{2}$ and a total ionizing dose of $12 \mathrm{MGy}$ are foreseen for the design integrated luminosity of $3000 \mathrm{fb}^{-1}$. A map of the expected particle fluence in the tracker after an integrated luminosity of $3000 \mathrm{fb}^{-1}$ is shown in Fig. 2. These values are about one order of magnitude above the design parameters used for the current detector. For this reason the CMS detector needs to undergo an overall upgrade in order to be fully functional in the new environment. In particular, the Inner Tracker, which will be the innermost part of the detector, need to be completely replaced in order to withstand the high radiation levels of the HL-LHC.

The most important requirements that are driving the design for the upgrade of the CMS Inner Tracker are:

- Radiation tolerance: the tracker has to be fully efficient up to the total integrated luminosity foreseen for the operational period of the HL-LHC. The Inner Tracker, which will house 


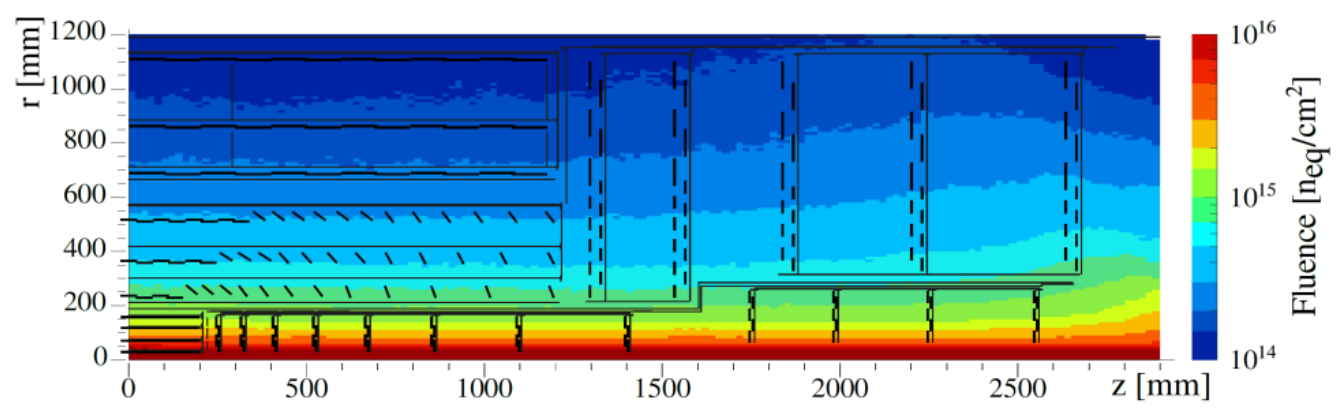

Figure 2: Map of the expected particle fluence in the Phase-2 CMS tracker after a total integrated luminosity of $3000 \mathrm{fb}^{-1}$. The expected fluence is strongly dependent on the radial coordinate and shows only a mild dependence along the $z$ coordinate [2].

the pixel detector, is designed to have the possibility to extract it during the shutdowns for maintenance and possible replacements of damaged modules.

- Extended acceptance: an extended coverage in the forward pseudorapidity region, up to $|\eta|=$ 4, will allow an efficient mitigation of the pileup and will improve the vertex reconstruction efficiency.

- Increased granularity: a high granularity will guarantee an occupancy as low as the per mille level under the high pileup conditions and efficient tracking performance especially in the reconstruction of high energy jets.

- Reduced material budget: a lighter tracker will improve the tracking performance, limiting the effects of hadronic interactions and multiple scattering.

\section{Inner Tracker detector layout}

The Phase-2 Inner Tracker is designed to maintain or improve the tracking and vertexing performance of the current detector, but in the high pileup scenario of HL-LHC. The current baseline for the layout of the Inner Tracker is shown in Fig. 3. The Inner Tracker, which is completely composed of pixel modules, comprises a cylindrical barrel part with four layers, referred to as Tracker Barrel Pixel Detector (TBPX), eight small double-discs per side, referred to as Tracker Forward Pixel Detector (TFPX), and four additional double-discs per side with larger radii, referred to as Tracker Endcap Pixel Detector (TEPX).

In TBPX the layers are placed at radii extending from $2.9 \mathrm{~cm}$, right outside the beam pipe, to $r=16 \mathrm{~cm}$, while they extend up to $\left|z_{\max }\right|=20 \mathrm{~cm}$ along the beam direction. The pixel modules in TBPX are arranged in ladders staggered in radius to obtain a projective overlap along $r-\phi$, and with no overlap along $z$. A projective gap at $\eta=0$ is avoided by mounting an odd number of modules along $z$. The first disk of TFPX is placed at $|z|=25 \mathrm{~cm}$, and the eighth disk at $|z|=140 \mathrm{~cm}$. The modules in each disk are arranged in concentric rings, obtaining an overlap in $r$ and $r-\phi$ because of the double-disc structure. For TEPX the discs are mounted between $|z|=175 \mathrm{~cm}$ and $|z|=255 \mathrm{~cm}$, and the modules are arranged similarly to TFPX. In order to facilitate the installation, each doubledisc of TFPX and TEPX is made of two "D"-shaped carbon fiber supports that hold the modules. 


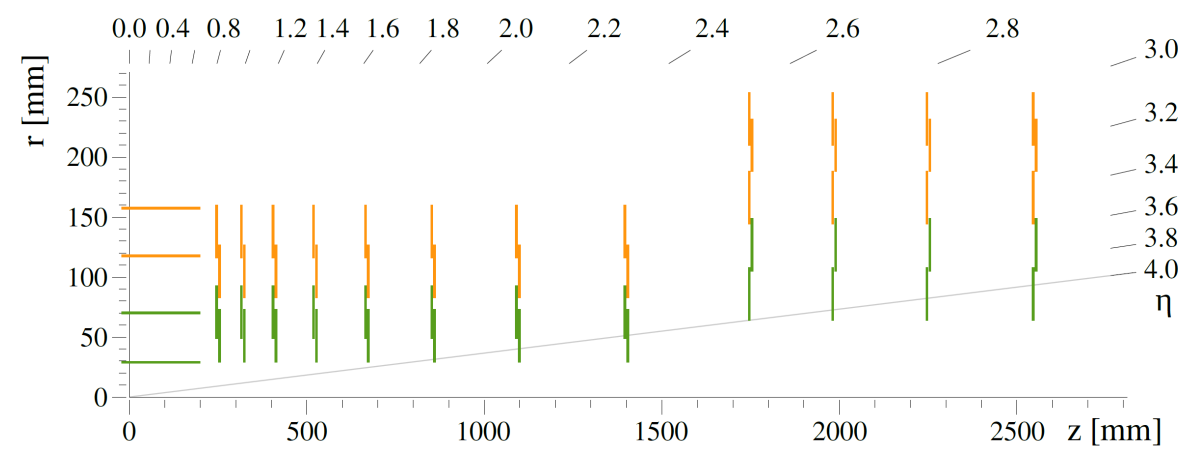

Figure 3: Sketch of one quarter of the pixel detector layout in the $r-z$ view. Green lines correspond to modules made of two readout chips and orange lines represent larger modules with four chips.

The pixel module is the basic unit of the Inner Tracker. It comprises a pixel sensor, several read-out chips (ROCs), a high-density flex circuit, and a mechanical support. Sensors are bumpbonded to the ROCs and glued to the flex circuits that, in turn, are wire-bonded to the ROCs. Heat generated on the modules is removed by using $\mathrm{CO}_{2}$ cooling pipes that keep the sensors and ROCs at an operational temperature of $-20^{\circ} \mathrm{C}$. The whole Inner Tracker will be equipped using only two different types of modules, comprising two or four ROCs, as shown in Fig. 4. The full Inner Tracker will consist of 4352 modules instrumenting a silicon area of about $4.9 \mathrm{~m}^{2}$.

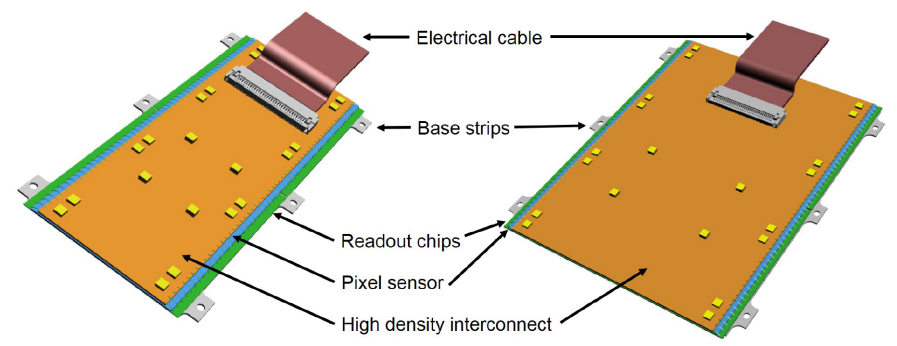

Figure 4: Sketch of the $1 \times 2$ (left) and $2 \times 2$ (right) pixel modules. The dimensions are roughly $1.8 \times 4.4 \mathrm{~cm}^{2}$ and $3.7 \times 4.4 \mathrm{~cm}^{2}$ for the $1 \times 2$ (left) and $2 \times 2$ modules, respectively.

\section{Sensors design parameters}

The Inner Tracker will be instrumented with small pitch radiation tolerant planar silicon sensors with the option of using three dimensional (3D) sensors [3] in the innermost region that is more exposed to radiation damage. The baseline design parameters for the planar pixel sensors are:

- $n$-on- $p$ substrate sensors produced on 6" silicon wafers. The $n$-on- $p$ technology has a lower cost with respect to $n$-on- $n$, due to the possibility of processing the wafer only on a single side. The main challenge of the $n$-on- $p$ technology is to prevent electric sparks in between the ROC, which is kept at ground potential, and the edge of the sensor. In fact, the high negative voltage that is applied on the backplane of the sensor can extend, in the absence of 
protections, along the edges eventually reaching the front face that is very close to the ROC. To prevent this, two protection systems are currently under study. The first one is a coating of the ROC wafer and of the sensor with a benzocyclobutene (BCB) layer, while the other is the post-process deposition of a Parylene layer on the full module.

- small thickness of the active area, between 100 and $150 \mu \mathrm{m}$. Under high radiation conditions, thin sensors are preferred over thick ones because the advantage of having a larger signal in thick sensors vanishes after the irradiation due to the severe trapping, which reduces the mean free path of charge carriers up to few tenths of microns, thereby limiting the charge collection efficiency. Moreover, thin sensors require a smaller bias voltage to reach the full depletion and generate a lower leakage current.

- small pitch pixels, with an area of $2500 \mu \mathrm{m}^{2}$ (six times smaller than the Phase-1 detector). Either rectangular or square aspect ratio is being considered, with sizes of $25 \times 100 \mu \mathrm{m}^{2}$ and $50 \times 50 \mu \mathrm{m}^{2}$, respectively. The realization of small cells is challenging, especially due to the limited space for $p$-stop and bias structures, and the design has to be optimized evaluating various options. The options considered for the pixel bias scheme, which is needed to test sensors before bump bonding, are punch through biasing common to several adjacent cells or the usage of temporary metals that are removed after testing. For the pixel isolation, both $p$-stop structures, possibly common to more cells, and $p$-spray deposition are being considered. Also, the usage of metal overhangs to mitigate large electric fields at the pixel implant edges is taken into account. The performance of the two aspect ratios have been compared using a Monte Carlo simulation of single muon tracks, processed through the CMS detector simulation and reconstruction software. This study takes into account only the TBPX part of the Phase-2 Inner Tracker and has been repeated for different sensor thicknesses, 100 and $150 \mu \mathrm{m}$, and three different signal thresholds, i.e. 1000, 1500 and 2000 electrons. Radiation damage effects are not taken into account in the simulation. The results of this study are shown in Fig. 5, where the spatial hit resolution for the first TBPX layer along the $z$-axis and in the $r \phi$ plane as a function of $\eta$ are reported. This study indicates that rectangular pixels perform better than square pixels in the transverse plane and have a comparable spatial resolution along the $z$-coordinate, especially in the $|\eta|>1$ region. Square pixels show a deterioration of the resolution in the scenario of large thickness and high threshold, which is likely due to a cluster breakage effect that occurs more often for inclined tracks.

Given the recent developments in 3D sensor technology, these devices have emerged as a potential option for the layers that are more exposed to radiation damage. 3D sensors are characterized by electrodes that penetrate the bulk, forming narrow columns. The depletion region extends in between adjacent columns and is therefore decoupled from the sensor thickness, allowing for thicker sensors to generate a larger signal while maintaining small depletion voltages and good charge collection efficiency. Given that the depletion region extension is smaller with respect to the planar sensors, the impact of charge carrier trapping is much reduced, thereby making 3D sensors radiation resistant devices. 


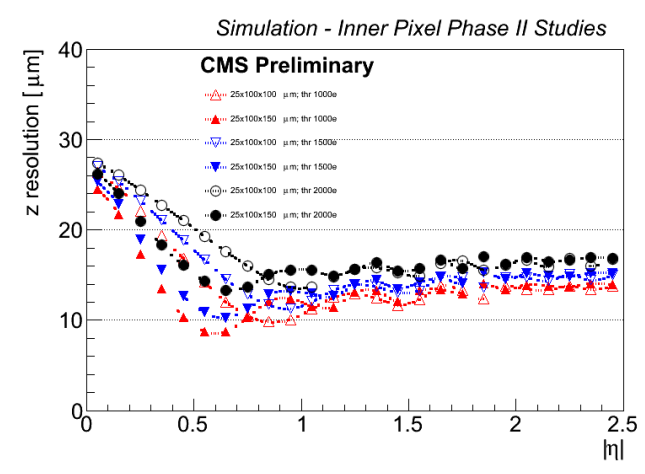

(a) $25 \times 100 \mu \mathrm{m}^{2}$

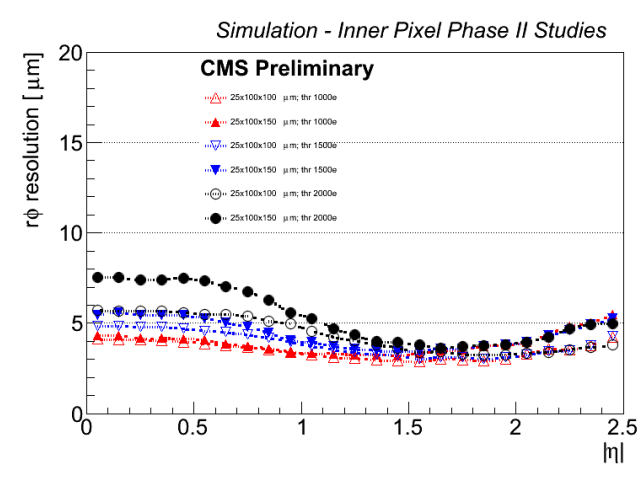

(c) $25 \times 100 \mu \mathrm{m}^{2}$

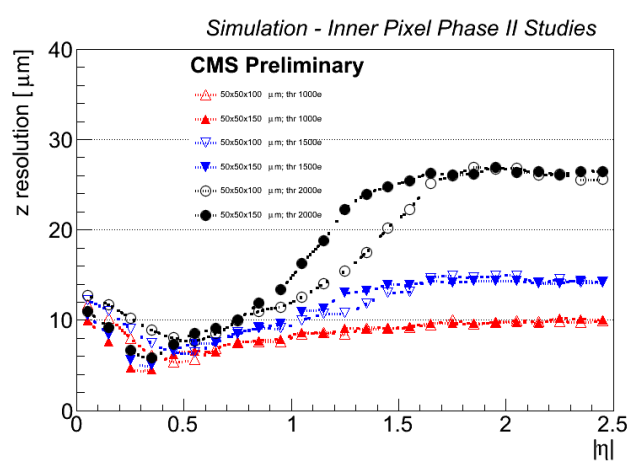

(b) $50 \times 50 \mu \mathrm{m}^{2}$

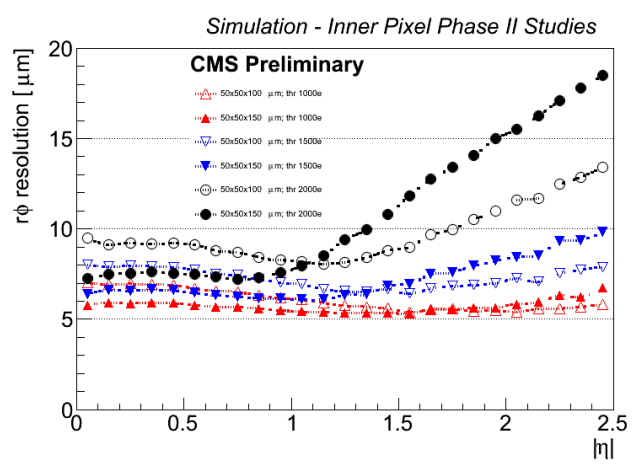

(d) $50 \times 50 \mu \mathrm{m}^{2}$

Figure 5: Hit resolution along the $z$-axis (top row) and in the $r-\phi$ transverse plane (bottom row) as a function of $\eta$ for pixels with $25 \times 100 \mu \mathrm{m}^{2}$ and $50 \times 50 \mu \mathrm{m}^{2}$ aspect ratio. Different colours correspond to different signal thresholds: 1000 e (red), 1500 e (blue), and 2000 e (black). Open and fileld markers refer to sensors with $100 \mu \mathrm{m}$ and $150 \mu \mathrm{m}$ active thickness, respectively.

\section{First R\&D results}

CMS has initiated extensive R\&D submissions to study the design options of thin planar and 3D devices. The batches of sensors have been produced by four different vendors: Hamamatsu Photonics, FBK, Sintef and CNM. The batches include sensors compatible with the present ROCs like FE-I4 [4] for ATLAS and PSI46dig [5] for CMS, or with different prototype ROCs like the new RD53A developed in $65 \mathrm{~nm}$ technology [6]. The main features of each submission are summarized in Table 1. While the production of the Hamamatsu batch is still ongoing at the time of writing, some highlights of FBK, Sintef and CNM submission results will be shown in this document. Sensors from these submissions are studied in test beam experiments, before and after irradiation campaigns performed in different facilities. All the studies have been performed using the PSI46dig ROC [5] (pixel size $100 \times 150 \mu \mathrm{m}^{2}$ ).

Thin $n$-on- $p$ pixel sensors with 100 and $130 \mu \mathrm{m}$ active thickness were produced by FBK Italy [7], and studied in a test beam campaign at FNAL $(120 \mathrm{GeV}$ protons at the FNAL MTest facility [8]). The collected charge was measured before and after irradiation with $800 \mathrm{MeV}$ protons at Los Alamos to $1.2 \times 10^{15} \mathrm{n}_{\mathrm{eq}} / \mathrm{cm}^{2}$. The distribution of the collected charge before and after irra- 
Table 1: Summary of R\&D planar and 3D sensor submissions for the Phase-2 Upgrade of the CMS Inner Tracker.

\begin{tabular}{|c|c|c|}
\hline Producer & Wafer characteristics & Goals \\
\hline \multirow[t]{4}{*}{ Hamamatsu Photonics } & $n$-on- $p$ & Square pixels vs. rectangular ones \\
\hline & Active thickness: $150 \mu \mathrm{m}$ & $p$-stop vs. $p$-spray isolation \\
\hline & & Bias scheme \\
\hline & & Metal overhang \\
\hline \multirow[t]{4}{*}{ FBK } & $n$-on- $p$ & Small pitch pixels \\
\hline & Active thickness: $100-130 \mu \mathrm{m}$ & Punch-through biasing scheme \\
\hline & & Spark protection using $\mathrm{BCB}$ \\
\hline & & Different wafer thinning procedures \\
\hline \multirow[t]{2}{*}{ Sintef } & $n$-on- $n$ & Slim edge pixels \\
\hline & Active thickness: $300 \mu \mathrm{m}$ & Small pitch pixels \\
\hline \multirow[t]{3}{*}{ FBK 3D } & $n$-on- $p$ & Small pitch pixels on thin substrate \\
\hline & Active thickness: $150 \mu \mathrm{m}$ & \\
\hline & Single sided DRIE process & \\
\hline \multirow[t]{3}{*}{ CNM 3D } & $n$-on- $p$ & Small pixels sizes \\
\hline & Active thickness: $150 \mu \mathrm{m}$ (single sided) & Slim edge pixels \\
\hline & Active thickness: $200-230 \mu \mathrm{m}$ (double sided) & \\
\hline
\end{tabular}

diation is reported in Fig. 6, showing that thin sensors provide a charge collection efficiency after irradiation consistent with other measurements obtained using $100 \mu \mathrm{m}$ thick strip sensors [2].

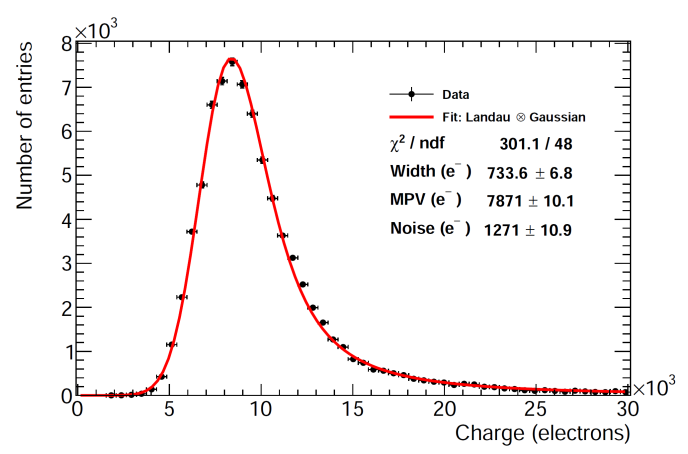

(a) Before irradiation

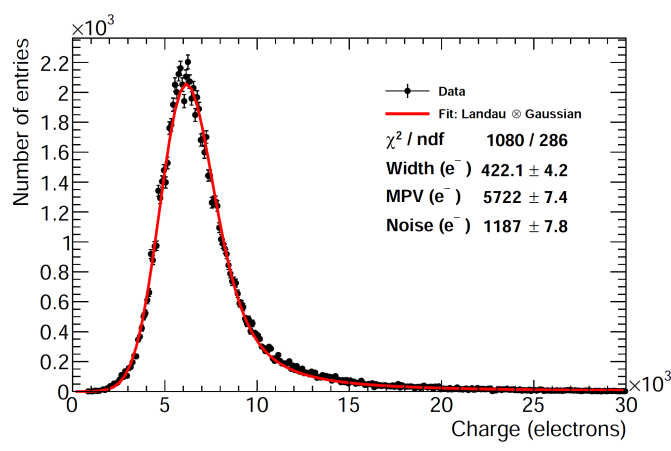

(b) After irradiation

Figure 6: Collected charge using single pixel clusters for an $n$-on- $p 130 \mu \mathrm{m}$ thick pixel sensor before (a) and after (b) irradiation. Before irradiation, the sensor was biased at $40 \mathrm{~V}$ and operated at $+25^{\circ} \mathrm{C}$. After irradiation, it was biased at $300 \mathrm{~V}$ and cooled to $-20^{\circ} \mathrm{C}$.

Small pitch sensors were studied by using sensors belonging to a Phase- 1 wafer submission by Sintef ( $n$-in- $n, 300 \mu \mathrm{m}$ thickness). The pixel size was $25 \times 600 \mu \mathrm{m}^{2}$, compatible with the PSI46dig ROC using a special bump bond pattern. Results on these sensors have demonstrated the feasibility of very small pixel cells [9]. Sintef submission has also been used to study slim edge sensors, in which the inactive edge of the pixel sensor (inefficient due to the presence of guard ring structures) was reduced decreasing the distance of the last pixel from the dicing edge down to $210 \mu \mathrm{m}$ ( $1150 \mu \mathrm{m}$ for the Phase- 1 sensors). The tests performed on these sensors showed that the inactive area can be reduced significantly, in spite of a reduction of the charge collected at the edge of the pixel due to the smaller guard ring structure [9]. 
Test 3D sensors have also been produced by FBK and CNM [10] and studied within CMS [11]. FBK produced sensors using a single-sided DRIE (Deep Reactive Ion Etching) technology to etch the columns in the high resistivity bulk of the wafer, while CNM produced 3D sensors using both single- and double-sided technologies. Schematics of 3D sensors produced using the two technologies are shown in Fig. 7.
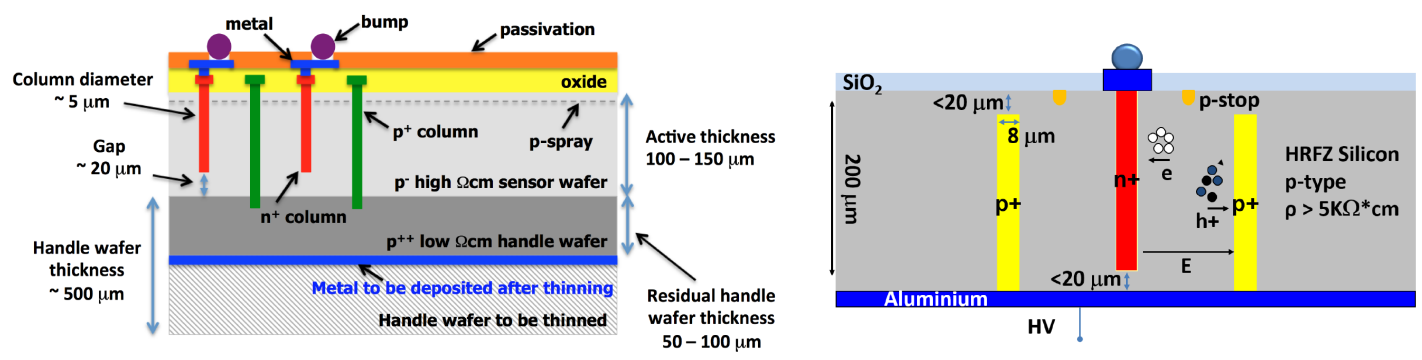

Figure 7: Schematics of 3D sensors produced by FBK (left) [12] and CNM (right) [10] using the singlesided and double-sided processes.

The FBK submission is focused on thin 3D sensors, testing different layouts of the pixel unit cell containing readout and ohmic columns. As an example, the option of using two electrodes instead of one in a $25 \times 100 \mu \mathrm{m}^{2}$ size cell would lead to a more radiation resistant design, due to the smaller distance between readout and ohmic columns. This is at the expenses of a dense layout that requires a well controlled production process. First 3D pixel sensors with $100 \times 150 \mu \mathrm{m}^{2}$ size and $130 \mu \mathrm{m}$ thickness have been studied in a test beam at the FNAL MTest facility. Figure 8 shows the cell hit efficiency map for one of the 3D pixel sensors with two readout columns for each pixel unit cell, as a function of the position of the beam in the pixel cell, obtained superimposing all pixel cells. The efficiency map is shown both for zero and 5 degrees tilting angle of the sensor with respect to the beam axis. While the left plot shows mild inefficiencies when the particles hit the readout and ohmic columns, the full efficiency (above 99\%) is recovered with a 5 degrees tilting angle.
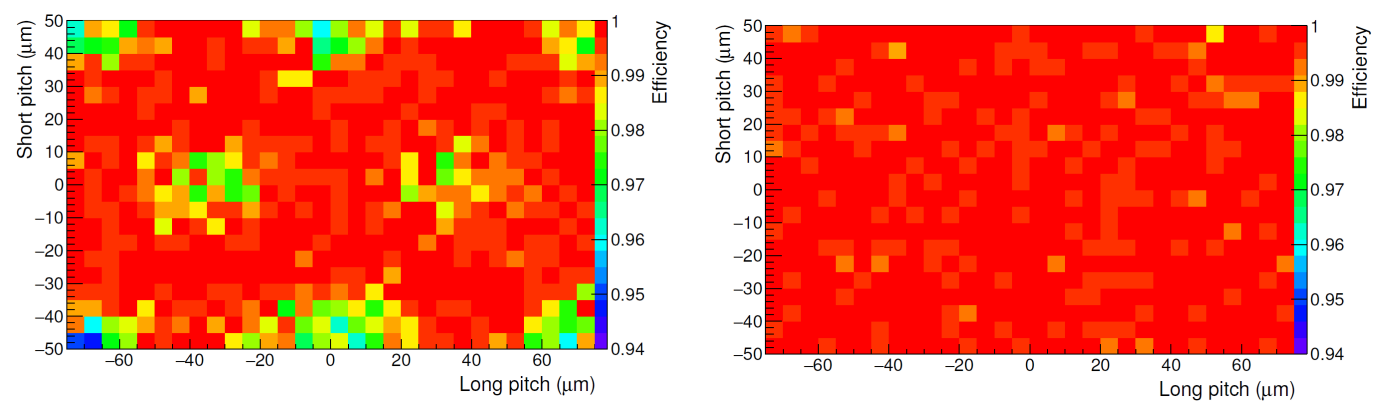

Figure 8: Cell hit efficiency measured at the FNAL test beam for an FBK 3D sensor with $100 \times 150 \mu \mathrm{m}^{2}$ pixel size and $130 \mu \mathrm{m}$ active thickness, for 0 degrees (left) and 5 degrees (right) tilting angle with respect to the beam axis. The sensor was biased at $30 \mathrm{~V}$ and operated at a temperature of $25^{\circ} \mathrm{C}$. The signal threshold in this measurement was of about 2000 electrons.

Small pitch sensors $\left(50 \times 50 \mu \mathrm{m}^{2}\right.$ and $\left.25 \times 100 \mu \mathrm{m}^{2}\right)$ with different pixel cell layouts were also tested. Since they were connected to the PSI46dig ROC, only one pixel cell over six was bump 
bonded to the ROC channels and read out, while the non read out cells were shorted together. The collected charge for a $50 \times 50 \mu \mathrm{m}^{2}$ pixel size sensor, with one readout column for each pixel unit cell, is shown in Fig. 9(a) for a bias voltage of $30 \mathrm{~V}$.

CNM produced special $230 \mu \mathrm{m}$ thick 3D sensors consisting of rows of small pixels with size of $25 \times 100 \mu \mathrm{m}^{2}$. Each sensor has 128 strips each consisting of 75 pixels shorted by aluminium connection along the long pitch. This design permits irradiating the sensor before the wire bonding to the ROC, therefore making it possible to measure the collected charge of highly irradiated devices using the ALIBAVA readout system [13]. The sensor was irradiated with $24 \mathrm{GeV}$ protons at the IRRAD $(C E R N)$ facility to a fluence of $5.7 \times 10^{15} \mathrm{n}_{\mathrm{eq}} / \mathrm{cm}^{2}$, and tested at the SPS test beam line at CERN using a mixed beam of $120 \mathrm{GeV}$ pions and protons. The measured charge distribution at a bias voltage of $180 \mathrm{~V}$ is shown in Fig. 9(b), and the charge collection efficiency was measured to be $94 \%$ of that of a non-irradiated device operated at $30 \mathrm{~V}$, therefore demonstrating the good performance of 3D sensors after irradiation.
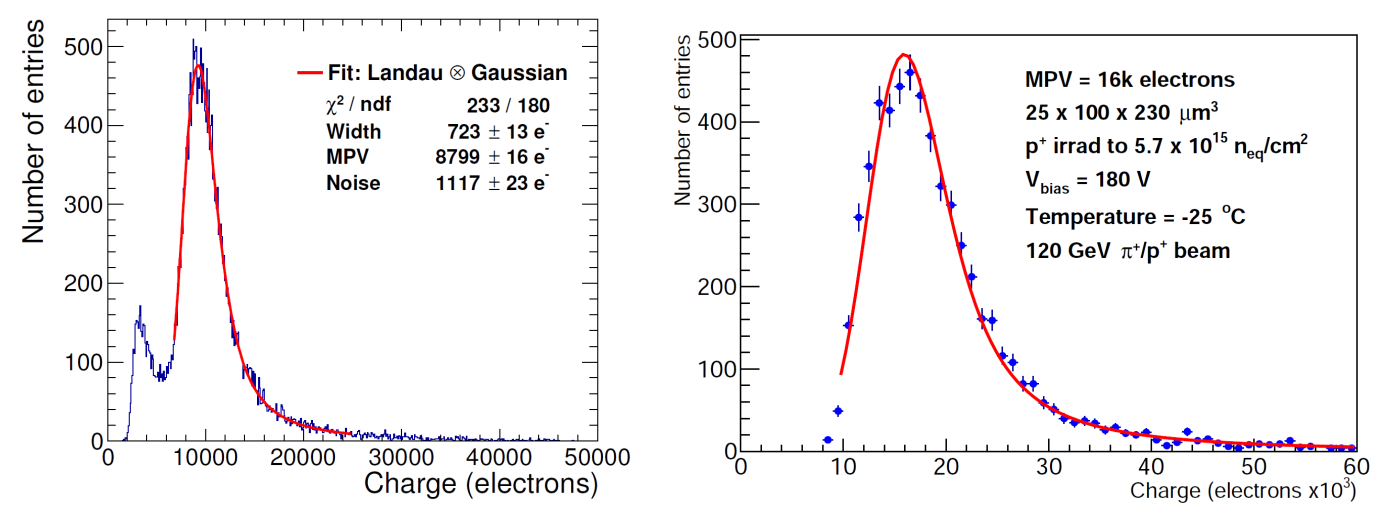

Figure 9: (Left) Collected charge distribution for a 3D sensor produced by FBK with $50 \times 50 \mu \mathrm{m}^{2}$ pixels. The second peak for cluster charges below 5000 electrons is an artefact due to charge shared by non read out adjacent pixels. (Right) Collected charge distribution for an irradiated 3D sensor produced by CNM with $25 \times 100 \mu \mathrm{m}^{2}$ pixels connected to from strips. Red lines represent the fit to a Landau distribution convoluted with a Gaussian.

\section{Conclusions}

The CMS detector will undergo an extended upgrade program for the HL-LHC phase. The current pixel detector will be replaced with a new Inner Tracker detector that will have good tracking and vertexing performance under the demanding conditions of the HL-LHC. In particular the new Inner Tracker will have to withstand radiation fluences up to $2.3 \times 10^{16} \mathrm{n}_{\mathrm{eq}} / \mathrm{cm}^{2}$ in the innermost layers and will have an extended acceptance up to $|\eta|=4$. A dense R\&D program is started within CMS to study the design of new pixel sensors with small active area and fine pitch cells. Thin $n$-on- $p$ planar sensors are the baseline option to instrument the whole tracker, with the viable option of using 3D sensors in the innermost layers. The first studies on both planar and 3D sensors have demonstrated the feasibility of thin sensors with small pixel cells, showing that these sensors can reach good charge collection efficiencies after irradiation with a fluence of $5.7 \times 10^{15} \mathrm{n}_{\text {eq }} / \mathrm{cm}^{2}$. 


\section{References}

[1] G. Apollinari et al., "High-Luminosity Large Hadron Collider (HL-LHC): Technical Design Report V. 0.1”, CERN-2017-007-M, doi http://dx.doi.org/10.23731/CYRM-2017-004

[2] CMS Collaboration, "The Phase-2 Upgrade of the CMS Tracker”, Technical Design Report CERN-LHCC-2017-009, CMS-TDR-014, 2017

[3] S. I. Parker et al., "3D - A proposed new architecture for solid-state radiation detectors", Nucl. Instrum. Meth. A 395 (1997) 328-343. doi 10.1016/S0168-9002(97)00694-3

[4] M. Barbero et al., "The FE-I4 Pixel Readout Chip and the IBL Module”, PoS (Vertex 2011) 038 (2011). doi 10.22323/1.137.0038

[5] H.C. Kästli, "Frontend electronics development for the CMS pixel detector upgrade", Nucl. Instrum. Meth. A 731 (2013) 88.

[6] RD53 Collaboration, "RD Collaboration Proposal: Development of pixel readout integrated circuits for extreme rate and radiation", Scientific Committee Paper CERN-LHCC-2013-008, LHCC-P-006, 2013.

[7] M. Meschini et al., "The INFN-FBK pixel R\&D program for HL-LHC” Nucl. Instrum. Meth. A 831 (2016) 116-121. doi 10.1016/j.nima.2016.05.009

[8] M. Dinardo et al., "The pixel tracking telescope at the Fermilab Test Beam Facility" Nucl. Instrum. Meth. A 811 (2016) 162-169. doi 10.1016/j.nima.2015.12.003

[9] C. Vernieri et al., "Pixel Sensors with slim edges and small pitches for the CMS upgrades for HL-LHC”, Nucl. Instrum. Meth. A 845 (2017) 189-193. doi 10.1016/j.nima.2016.06.020

[10] G. Pellegrini et al., "First double-sided 3-D detectors fabricated at CNM-IMB", Nucl. Instrum. Meth. A 592 (2008) 38. doi 10.1016/j.nima.2008.03.119

[11] I. Zoi et al., "Beam Test Results of Thin n-in-p 3D and Planar Pixel Sensors for the High Luminosity LHC Tracker Upgrade at CMS”, PoS (EPS-HEP2017) 809 (2017). doi 10.22323/1.314.0809

[12] G. F. Dalla Betta et al., "Development of a new generation of 3D pixel sensors for HL-LHC” Nucl. Instrum. Meth. A 824 (2016) 386-387. doi 10.1016/j.nima.2015.08.032

[13] ALIBAVA Systems. http://www.alibavasystems.com 